\title{
Current management of pelvic fracture urethral injuries: to realign or not?
}

\author{
Ashley N. Dixon, Jack C. Webb, Jessica L. Wenzel, J. Stuart Wolf Jr, E. Charles Osterberg \\ Dell Medical School at The University of Texas, Austin, TX, USA \\ Contributions: (I) Conception and design: All authors; (II) Administrative support: All authors; (III) Provision of study materials or patients: None; \\ (IV) Collection and assembly of data: AN Dixon; (V) Data analysis and interpretation: All authors; (VI) Manuscript writing: All authors; (VII) Final \\ approval manuscript: All authors. \\ Correspondence to: E. Charles Osterberg. Dell Medical School at The University of Texas, Austin, TX 78701, USA. Email: Charles.osterberg@austin.utexas.edu.
}

\begin{abstract}
The acute management of pelvic fracture urethral injuries (PFUIs) remains a controversial topic. Currently, suprapubic tube (SPT) placement with delayed repair or primary realignment (PR) represents the strategies used to treat patients. While many will advocate the use of one technique over the other, the 2014 American Urological Association (AUA) Guidelines give providers the option for the management PFUI. Current literature evaluates these two interventions, focusing on the incidence of re-stricture formation, erectile dysfunction, and urinary incontinence. Here we perform a comprehensive review of the current management for PFUI, as well as, discuss the limitations of the studies and need for more prospective studies on this debated topic.
\end{abstract}

Keywords: Cystostomy; endoscopic realignment; pelvic fracture; urethral stricture; urethral trauma; pelvic fracture urethral injury (PFUI)

Submitted Dec 01, 2017. Accepted for publication Jan 18, 2018.

doi: $10.21037 /$ tau.2018.01.14

View this article at: http://dx.doi.org/10.21037/tau.2018.01.14

\section{Clinical case}

A 35 year-old male was brought in by ambulance following a high-speed motor vehicle collision. Primary survey revealed an unstable pelvis, hypotension, and blood at the meatus. Initial attempt at Foley catheter placement was unsuccessful. A retrograde urethrogram revealed a complete pelvic fracture urethral injury (PFUI) with complete disruption of the prostatomembranous urethra and no contrast entering the bladder. Should the urologist attempt primary endoscopic realignment (PER) or simply place a suprapubic tube (SPT) and plan for delayed posterior urethroplasty? In this review, we present the most current data that supports PER $v s$. SPT and delayed reconstruction.

\section{Introduction}

PFUIs most commonly result from the shearing forces to the bulbo-membranous junction causing the urethra to avulse from its position within the urogenital diaphragm. Less frequently, they are caused by pelvic bony fragment laceration, or pubic symphysis diathesis (1). The current incidence of PFUI is noted to be between $5-25 \%$ and is usually the result of blunt injuries sustained in motor vehicle accidents (2-5). Many of the reported urethral injuries occur in men, as a PFUI in women is rare $(<1 \%)$ because of shortened urethral length, mobility, and lack of attachments to the pubic symphysis $(2,6)$. Urologists and other physicians should have a high degree of suspicion of a PFUI if a patient presents with the following: pelvic trauma, blood at the meatus, inability to void, hematuria and dysuria, scrotal, labial, perineal, and/or penile swelling, a highriding or impalpable prostate, and difficulty/inability to insert a catheter (1). The first step in assessing a patient for a PFUI is to perform a retrograde urethrography, which is considered the preferred diagnostic modality (7). However, if the urethral injury is thought to have occurred secondary to a penile fracture, then urethroscopy can be used instead (7). 
The management of PFUIs is a hotly debated and controversial topic. Pundits advocating for SPT or PER argue over which procedure produces the best outcomes in relation to erectile dysfunction, urinary incontinence, and re-stricture formation, as these issues can lead to a decreased quality of life in patients who were otherwise healthy prior to injury. The 2014 AUA Urotrauma Guidelines leave it up to interpretation as to whether SPT or PER is a superior management for PFUI (8). This article is meant to be a comprehensive review of the current literature available on the management of PFUI with SPT and delayed urethroplasty or PER.

\section{Methods}

We reviewed all of the current literature regarding PER and suprapubic cystostomy as a treatment for PFUI. A comprehensive summary of all the data was separated into levels of evidence (9). Level I evidence was considered to be a high-quality randomized control trial (RCT) or systematic review with adequate power. Level II was considered any poorly designed RCT, prospective cohort study, or systematic review of these studies. Level III studies were defined as retrospective cohort study, case-control study, or systematic review of level III studies. A level IV study was considered any case-series. After reviewing all of the current literature the highest level of evidence was determined to be level II and the lowest was level IV. No level V data was considered.

\section{Primary realignment (PR)}

Early realignment was first described in 1934 by Ormond and Cothran, and later by Wilkinson in 1961, as a treatment for acute urethral injuries $(10,11)$. Since that first introduction, several techniques, including: interlocking urethral sounds, retrograde catheter placement under direct vision, and combined anterograde/retrograde catheters have been described to re-establish early urethral realignment (12-15). However, these early attempts at realignment were considered unfavorable due to the highrisk of complications, impotence, and incontinence (12). Today, urethral alignment is performed by endoscopic guidance with urethroscopes. This is usually done with one cystoscope placed anterograde (through the suprapubic tract) and the other placed retrograde (through the external meatus). A wire is then passed through the anterograde scope, grasped with forceps via the retrograde scope, and externalized via the urethral meatus. Afterwards, the wire then acts as a guide for the placement of a Council tip Foley catheter, which is left in place for a range of 4 to 6 weeks (1). Advocates of the PER approach, have noted decreased incontinence and erectile dysfunction rates, a quicker time to spontaneous voiding, decreased need for urethroplasty and stricture formation. In addition, some orthopedic surgeons maintain that treatment with SPT increases the risk of hardware infection; although no literature exists that supports this claim (16). Opponents argue that PER just delays urethroplasty, and might not be the best choice for patients who are clinically unstable $(17,18)$. More recently, the cost-effectiveness of SPT vs. PER has been analyzed; with data showing PER to be more cost-effective with regards to management of complications compared to SPT $(19,20)$. Herein, we present data which supports PER or non-endoscopic realignment as independent management strategies for PFUI.

\section{Level 2 studies}

\section{Endoscopic realignment}

In the only level 2 study to date, Hadjizacharia et al. performed a prospective study of 21 patients with PFUI treated with either PER or SPT between 2000-2006 (16). The primary outcome measures were time to spontaneous voiding and urethral stricture rate. Eighteen patients were treated initially treated with PER, however this method failed in four, so they were treated with SPT and delayed urethroplasty. Three patients had no attempt at PER due to urologist preference and were thus treated with SPT. Therefore, after accounting for this 14 patients were treated with PER and 7 patients with SPT and delayed repair. The stricture rate in the PER group was $14 \%$ and $100 \%$ in the SPT group. There was no significant difference in time from initial treatment to stricture formation $(106 \pm 122$ days PER vs. $139 \pm 81$ days SPT; $\mathrm{P}=0.68)$ or the time from stricture development to last follow-up $(71 \pm 71$ vs. $220 \pm 87$ days; $\mathrm{P}=0.36)$. Two patients who were treated with PER developed a stricture afterwards, but did not require urethroplasty. One patient performed intermittent selfcatheterization for 1 month, and the other was treated with dilation for 2.5 months. The PER group had a statistically significant shorter time to spontaneous voiding compared to the SPT group $(35 \pm 23$ vs. $229 \pm 79$ days; $\mathrm{P}=0.002)$. In summary, this study showed that PER resulted in a significantly shorter time to spontaneous voiding and a decreased risk of urethral stricture. However, limitations 
included the small samples size and a cross-over design resulting in the comparison of PER and a control group of failed PER and SPT.

\section{Level 3 studies}

The majority of studies in support of realignment are level III studies, which are retrospective cohort studies and systematic reviews of other level III studies.

\section{Non-endoscopic PR}

In 1996, Koraitim reported the rates of stricture formation, urinary incontinence, and erectile dysfunction in 100 male patients with PFUI treated with either SPT (n=73), PR $(n=23)$, or primary suturing $(n=4)(21)$. PR was performed with interlocking sounds using a sound-to-sound or soundto-finger method to re-form urethral continuity over the catheter. Primary suturing was performed at the time of injury by anastomosing both ends of the disrupted urethra around a Foley catheter. In 71 patients treated with SPT, $97 \%$ failed urethral voiding and ultimately underwent a delayed endoscopic realignment. This was the same for $12 / 23$ patients (52\%) treated with $\mathrm{PR}$ and $2 / 4$ patients $(50 \%)$ treated with primary suturing. Incontinence developed in $2.7 \%$ of patients in the SPT group, $4 \%$ treated with PR, and $0 \%$ in the primary suturing group. After excluding children, it was noted that in patients who were potent prior to injury, 9/50 (18\%), 5/18 (28\%), and 2/4 (50\%) became impotent in the SPT, PR, and primary suturing groups respectively. The incidence of stricture formation was decreased by $52 \%$ in the PR and $50 \%$ in the primary suturing group. However, the incidence of impotence increased by $28 \%$ (PR) and 50\% (primary suturing). In conclusion, SPT had a higher incidence of stricture formation and PR and primary suturing were more likely to result in impotence.

Asci et al. published a retrospective cohort study in 1999 with 38 male patients managed with either SPT $(n=18)$ or PR $(n=20)$ with the primary aim of determining voiding and sexual dysfunction and evaluation of urethral stricture reformation (22). Urethral stricture rate was found to be statistically significant (83.3\% SPT vs. 45.0\% PR; P=0.014). There was no significance difference between impotence (17.6\% SPT vs. 20\% PR; P=0.855) and incontinence (5.6\% SPT vs. $10.0 \%$ PR; P=0.611). Ultimately, $8 / 18$ (44\%) of patients treated with SPT and 2/20 (10\%) treated with PR underwent urethroplasty and this was statistically significant $(\mathrm{P}=0.016)$. Therefore, the authors concluded that $\mathrm{PR}$ does not result in significant impact on impotence or incontinence and could prevent need for complex urethroplasty.

A retrospective study done by Mouraviev et al. evaluated the incidence of stricture, impotence, and incontinence in patients treated with PR and SPT. Urethral stricture occurred in $100 \%$ of SPT patients and 49\% PR patients (23). All patients who presented with stricture were treated with direct visual internal urethrotomy (DVIU) one time. DVIU was successful in 49\% undergoing PR and $47 \%$ SPT $(\mathrm{P}<0.05)$ and PR patients on average required 1.6 DVIU procedures compared to 3.1 for those treated with SPT. Ultimately, 24\% (PR) and 47\% (SPT) required urethroplasty for their stricture. Of the patients treated with $\mathrm{PR}, 34 \%$ developed impotence and 18\% incontinence vs. $42 \%$ impotence and $26 \%$ incontinence in those managed with SPT $(\mathrm{P}<0.05)$. To summarize, the authors were proponents of $\mathrm{PR}$ for management of PFUI due to the lower risk of stricture, stricture complexity, and fewer DVIU procedures.

\section{PER}

Johnsen et al. performed a retrospective cohort study, which identified 41 patients between 2000-2014 who presented with traumatic PFUIs at their institution (24). Of the 41 patients, 27 were managed with PER and 14 with SPT. The primary aim of the study was to determine success of PER. Failure was defined as symptomatic stricture requiring intervention. After catheter removal 10/27 (37\%) PER patients required no further intervention. PER failed in 17 of 27 patients (63\%). Of the PER failures, 10/17 were managed with chronic intermittent catheterization or DVIU. Those undergoing DVIU underwent on average 1.2 endoscopic interventions (median 1, range, 1 to 2 ) over a mean 51 months follow-up (median 37.6 months, range, 6 to 138 months). Urethroplasty was done in $7 / 27$ (25.9\%) PER patients and 11/14 (78.6\%) SPT patients $(\mathrm{P}=0.0023)$. It was noted that time from initial injury to urethroplasty was significantly shorter in the SPT group compared to the PER group (mean $5.8 \pm 1.6$ vs. $14.6 \pm 7.6$ months; $\mathrm{P}=0.003$ ). Limitations of this study were that patients were referred from other institutions or treated with SPT by another surgical team and the small cohort size. Following this study, the authors concluded that while PER is a definitive treatment for a third of patients, half of those who fail have a prolonged time to urethroplasty and thus require longer follow-up and possibly more interventions.

Barrett et al. performed a systematic review and metaanalysis comparing PER and SPT in patients with PFUI. 
The primary aim of the study was to assess for the rates of stricture formation, erectile dysfunction, and incontinence. Nine studies were included for a total of 464 patients (PER =216 vs. SPT =248). Stricture rate was found to be significantly lower in the PER group $(\mathrm{OR}=0.12 ; 95 \%$ CI: $0.04-0.41 ; \mathrm{P}<0.001)$ with a number needed to treat of 2.76 to prevent one urethral stricture (25). There was no significance seen in regards to erectile dysfunction and incontinence, between PER $v s$. SPT, respectively (OR $=1.19,95 \%$ CI: $0.73-1.92, \mathrm{P}=0.49$; OR $=0.75,95 \%$ CI: $0.38-1.48, \mathrm{P}=0.41$ ).

A recent study on PER by Chang et al. who performed a retrospective review of patients treated with either SPT and delayed realignment or PER, with the primary aim of evaluating the cost-effectiveness of each procedure over a 2 -year period (20). Twenty-two patients (SPT $=11, \mathrm{PER}=11$ ) were evaluated, and following initial treatment 21/22 (95\%) developed a recurrent urethral stricture. Only one patient from the PER group was stricture free. Over the span of 2 years, the SPT group required more internal urethrotomies then the PER group (4.1 vs. 1.8, $\mathrm{P}=0.009$ ) for urethral strictures. The cost for the initial management for the PER group was 3 times higher than that of the SPT group (\$921.84 vs. \$265.43). However, by the end of the first year the costs for SPT was higher $(\$ 2279.64$ SPT $v$ s. $\$ 1893.84$ PER; $\mathrm{P}=0.493$ ) and by the end of the second year (\$3475.99 PER vs. \$2267.70 SPT; P=0.015) a statistically significant difference was seen. In conclusion, the authors noted a decrease need for urethrotomy procedures in patients treated with PER as well as decreased economic costs after 2 years from initial injury.

Koraitim et al. retrospectively reviewed the medical records of 94 patients referred from an outside institution from 1995 to 2009 (26). They analyzed initial management of urethral injuries, length of urethral defect, and type of delayed repair, incontinence, and impotence. Fortytwo patients were treated with PER and 52 patients with SPT. Realignment was performed within 24 hours of presentation. The mean length of the urethral gap was considered statistically significant and noted to be $2.0 \mathrm{~cm}$ (range, 0.5-5.5 cm) for PER and $2.5 \mathrm{~cm}$ (range, 0.9-6.0 cm) for SPT $(\mathrm{P}<0.05)$. Patients initially managed by delayed repair using a simple perineal approach $32 / 42,76 \%$ PER vs. $30 / 52,58 \%$ SPT; $\mathrm{P}<0.05)$ and a combined perineoabdominal procedure (10/42, 24\% PER vs. 22/52, $42 \%$; $\mathrm{P} \leq 0.05)$ were more frequently corrected than by those undergoing SPT. Urinary incontinence was reported in one patient managed with PER which was secondary to a pelvic fracture injury of the bladder neck. Erectile dysfunction was noted in 10/42 (24\%) patients undergoing PER and 2/52 (4\%) treated with SPT. To summarize, the authors concluded that although PER is not superior to SPT it significantly decreases the length of the urethral defect and thus indirectly facilitates delayed repair.

Zou et al. published a similar retrospective study in 2017, analyzing stricture reformation and length, intervention technique, and long-term functional outcome data on 522 patients treated with either PER or SPT from 2004-2014 (18). Mean time to PER was 3 days (range, $1-5$ days). Of the patients treated with PER, 18/129 (14\%) required no further treatments at a mean follow-up of 30 months (range, 21-77 months). Stricture developed in 111/129 (86\%) of PER patients. Average time to stricture formation was significantly higher in the PER group [23.5 months, (range, 4-34 months)] compared to the SPT group [7.6 months, (range, 3-14 months); $\mathrm{P}<0.05$ ]. The rate of management options significantly differed between groups (all, $\mathrm{P}<0.05$ ) and included internal urethrotomy, urethroplasty, urethral mobilization, inferior pubectomy, and urethral rerouting. Of note, the success rate of urethroplasty as well as the other procedures did not differ amongst PER and SPT (all, $\mathrm{P}>0.05)$. As in other studies, the authors advocated the use of PER in clinically stable patients as their data reflected less complex strictures and a decrease in patients requiring further intervention for their PFUI.

\section{Level 4 studies}

Here we present the level IV studies, which is a compilation of small case series.

\section{Non-endoscopic PR}

A small case series by Elliott et al. evaluated impotence, incontinence, and stricture formation in a total of 57 patients from 1950 to 1995 who were treated with PR (13). PR was performed retrograde urethral catheter placement $(56.1 \%)$, retrograde and antegrade catheter placement (15.7\%), or using interlocking sounds (29.8\%). Forty-two of 53 patients (79\%) reported normal erections compared to $7(13 \%)$ who reported a decreased quality of erection. However, it was noted that two of the patients reported having a decrease in quality of erections prior to injury. Normal urinary control was reported in 51/53 (96.2\%) of patients while $2 / 53(3.8 \%)$ reported mild stress urinary incontinence that did not require protective padding. A total of 18 patients (34\%) required no further urological 
intervention based on negative radiographic evidence or urethral stricture symptoms. Strictures developed in 36/53 (68\%) of patients, including $23(43 \%)$ with mild strictures who either did not require therapy or were treated with occasional in office dilation. More significant strictures developed in 13 patients $(25 \%)$ who were treated with the following: urethral sounds (8 cases), laser ablation of stricture (4 cases), DVIU (4 cases), transpubic urethroplasty (3 cases), and 1-stage urethroplasty (1 case). In summation, the authors found that PR was procedure of choice for stable patients being treated by a urologist with experience in PR.

\section{PER}

Among level IV studies for PER, 19 patients with PFUI from Harborview Medical Center were reviewed from 2004-2010. The primary aim of the study was to analyze the success of early (mean of 2 days) PER after blunt PFUI (27). Secondary aims were to assess incontinence and erectile dysfunction on follow-up visits. They defined failure of PER as patients requiring repeat procedures or self-catheterization after catheter removal. Using intent to treat analysis, the success rate of PER was $4 / 19$ (21.1\%). PER failed in $78.9 \%$ with a mean time to failure of 79 days. After PER failure, 11 patients went on to posterior urethroplasty with $3 / 11$ having already failed DVIU. None of the four patients treated successfully with PER reported incontinence or erectile dysfunction. Four patients whom PER failed reported erectile dysfunction but no incontinence issues. It was noted that these patients had a complete posterior urethral disruption. For the patients that reported erectile dysfunction issues, no standardized questionnaire was used for assessment. There was also no mention of if patients who presented with PFUI had erectile dysfunction or incontinence issues prior to their urethral injuries. In summary, it was concluded that patients being treated with PER had a high rate of stricture formation requiring operative intervention.

In 2001, Moudouni et al. retrospectively reviewed the charts of 29 men to determine the success rate and postoperative complications of early PER (28). All patients were initially treated with SPT and the average time to realignment was 3.8 days (range, 0-8 days). Potency was maintained in $25 / 29(86 \%)$ patients, urethral strictures developed in $12 / 29$ (41\%) with a mean time to stricture formation after PER of 6.2 months (range, 1.5-24 months). All patients were continent. Limitations of this study included a lack of hemodynamically instability reporting and no mention of how continence and potency were assessed. The authors concluded that early PER could lessen the complexity of urethral stricture and had minimal effect on erectile function and continence.

\section{Summary on PR and PER}

$\mathrm{PR}$ is a technique that has been around for the past 80 years. While, not initially considered the management option of choice due to its unacceptable rates of impotence and urinary incontinence, recent advances in endoscopes have introduced it back into the conversation as a viable and potentially superior management option for the treatment of PFUI. We present data supporting PR and PER with 14-66\% of patients at risk for re-stricture formation, $14-62.5 \%$ at risk for erectile dysfunction, and $4-25 \%$ of patients are at risk for urinary incontinence. See Table 1 for a summary.

Conversely, the studies presented were not without their limitations. As much of the literature is comprised of retrospective cohort and case series studies from referral centers, they lack long term follow up, standardized stricture surveillance strategies, gradation on the severity of the initial urethral injury, and standardized methods used for realignment. The heterogeneity and crossover between PER/PR groups vs. SPT group makes the results difficult to interpret. Furthermore, current data lacks a standardization of what is considered "early" or "immediate" realignment and usage of a validated tool for determining erectile dysfunction and incontinence. While, the current data does not suggest PER to be a superior option for the acute management of PFUI, in patients are clinically stable and at a hospital with a well-trained urologist, PER remains a is a viable option.

\section{Suprapubic cystostomy with delayed treatment}

In 1953, Johanson was the first to advocate the use of suprapubic cystostomy and delayed urethral repair (29). The general principle being that urine is diverted with a SPT and the urethral stricture that would inevitably develop is repaired with urethroplasty several months later. With this management strategy, no attempt is made to evacuate the hematoma or explore the retropubic space. Morehouse et al. noted several advantages to this technique: minimal fibrosis by allowing absorption of the hematoma and not exploring the retropubic space, easy feasibility in a poly-trauma patient, no danger of converting a partial tear into a complete tear, healing by secondary epithelialization if the defect is partial negating the need 


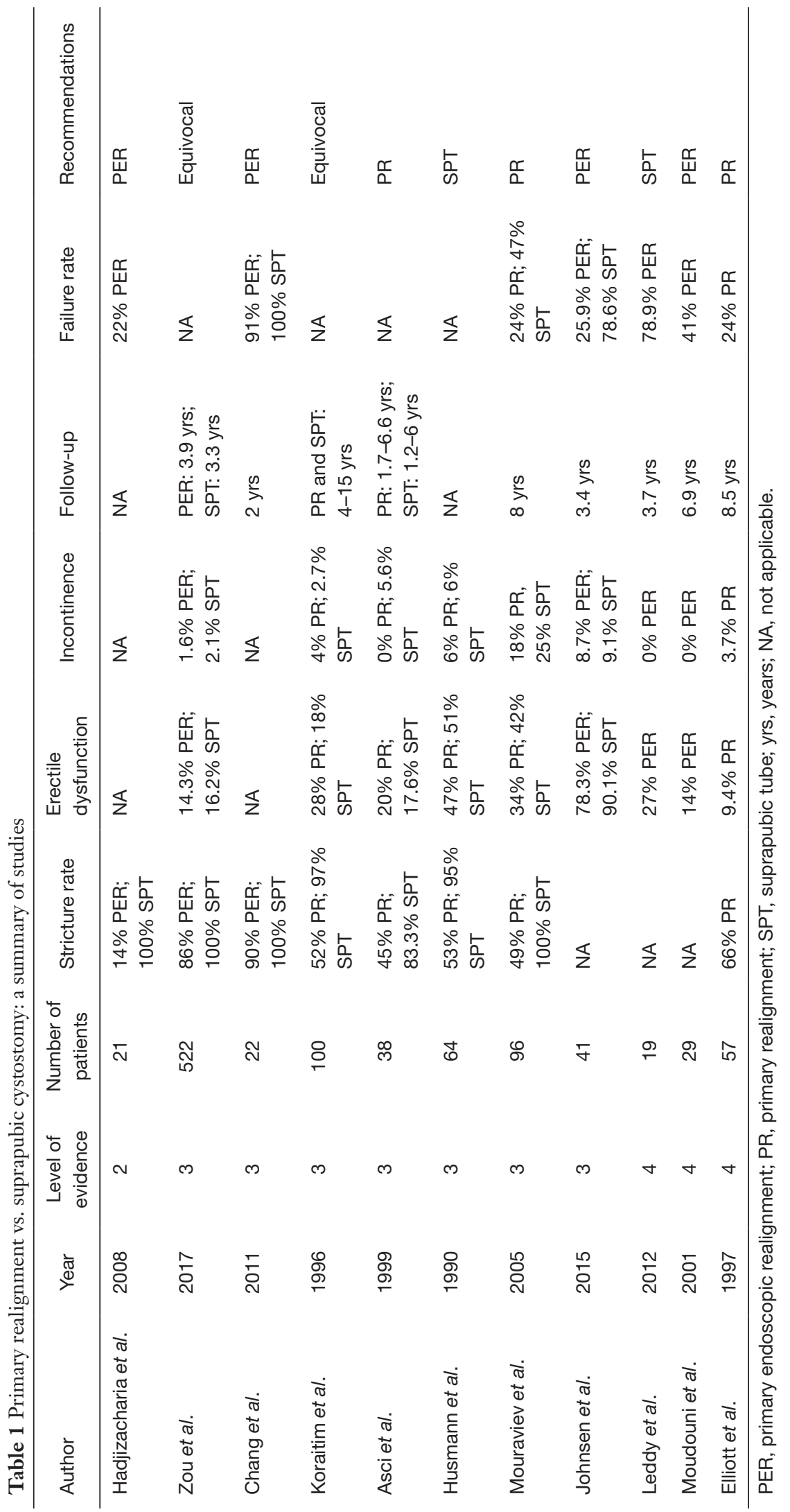


for urethral reconstruction, decreased incidence of erectile dysfunction, incontinence, and re-stricture formation following urethroplasty (30). SPT, however, does offer several disadvantages as long-term use can lead to increased risk of urinary tract infection, bladder calculi, and wound infection. Here we summarize current data in support of SPT and delayed urethroplasty following PFUI.

\section{Level 3 studies}

In 2015, Tausch and Morey analyzed the data of 40 patients who presented between 2007-2014 with PFUIs, and were treated with either SPT alone $(n=23)$ or PER $(n=17)(17)$. Their analysis focused on stricture length, urethroplasty technique, and time to symptom resolution, number of interval interventions, adverse events, and treatment outcomes. Patients undergoing SPT had a significantly shorter time to resolution of obstructive voiding [mean 6 months (range, 3-15 months)] than the PER group [mean 25 months (range, 4-574 months); $\mathrm{P}<0.01$ ]. Those treated with PER (10/17, 59\%) were 5 times more likely to have a delay of $>1$ year before urethroplasty compared to SPT. Statistical significance was also seen for patients of PER requiring several interval endoscopic procedures [median 4 (range, $1-36$ ); $\mathrm{P}<0.01$ ] prior to referral vs. none for SPT. The initial success rate was noted to be lower in the PER group (14/17, 82\%) compared to the SPT group $(23 / 23,100 \% ; \mathrm{P}<0.05)$. Adverse events, defined as failure of initial urethroplasty, the need for prolonged self-dilation ( $>6$ months), overflow incontinence, and pelvic abscess were noted to be higher in the PER group, however, no quantitative data for this was provided.

Another retrospective study, by Tausch et al. in 2014, analyzed the frequency of adverse events for patients undergoing PER ( $\mathrm{n}=17)$ and SPT $(\mathrm{n}=21)$ (31). Patients were stratified by their initial treatment prior to referral. Adverse events were determined to be the following: initial urethroplasty failures, prolonged self-dilation ( $>6$ months), overflow incontinence, and pelvic abscess formation prior to referral. Patients treated with PER had at least one or more adverse events $(2 / 21,10 \%$ SPT vs. $14 / 17,82 \%$ PER; $\mathrm{P}<0.05)$. This included more than 1 year to durable resolution $(2 / 21,10 \%$ SPT vs. 10/17, 59\% PER; $\mathrm{P}<0.05)$, failure of initial urethroplasty $(0 / 21,0 \%$ SPT vs. $3 / 17$, $18 \%$ PER; $\mathrm{P}<0.05$ ), and need for self-catherization for greater than 6 months $(0 / 21,0 \%$ SPT vs. $3 / 17,18 \%$ PER; $\mathrm{P}<0.05)$. There was no significant difference in overflow urinary incontinence $(0 / 21,0 \% \mathrm{SPT} v s .1 / 17,6 \%$ PER;
$\mathrm{P}=0.027)$ and pelvic abscess $(0 / 21,0 \% \mathrm{SPT}$ vs. $1 / 17,6 \%$ PER; $\mathrm{P}=0.027)$. A significant difference $(\mathrm{P}<0.01)$ was seen in the median interval endoscopic interventions between PER [median 4 (range, 1-36)] compared to none in those managed with SPT. It was also noted that PER patients had a lower urethroplasty success rate $(14 / 17,82 \%)$ compared to the SPT group $(21 / 21,100 \% ; \mathrm{P}<0.05)$. Following their study the authors concluded that PER did not facilitate easier urethroplasty and resulted in repeated endoscopic interventions.

Husmann et al. evaluated a group of 81 patients who suffered a urethral disruption secondary to PFUI. Sixtyfour patients were managed with SPT and 17 with PR (32). PR was performed using interlocking sounds. Outcomes were classified as: good (no urinary incontinence and further intervention), fair (1-2 internal urethrotomies or $\leq 4$ urethral dilations), and poor (impassable or recurrent strictures). Erectile dysfunction was determined by history alone. The authors found that $95 \%$ of patients in the SPT group developed impassable strictures of which 40 patients were treated with transpubic urethroplasty, 14 with perineal urethrotomy, and 7 with visual internal urethrotomy. The other three patients treated with SPT healed without any evidence of stricture formation. Good/fair results were obtained in $94 \%$ of patients treated with transpubic urethroplasty, $86 \%$ perineal urethroplasty, and $57 \%$ with visual internal urethrotomy. In comparison, in the PR group only $47 \%$ of patients had a satisfactory result (good or fair) after a median follow-up of 3 years (range, 3 months to 5 years). Furthermore, 9/17 (53\%) patients in the PR group developed impassable strictures refractory to urethrotomy and dilation, requiring urethroplasty for definitive repair. It was also noted that internal urethrotomies and PR had a significantly lower success rate when compared to urethroplasty $(\mathrm{P}<0.03$ and $\mathrm{P}<0.001$, respectively). Impotence was noted in $47 \%$ of the PR patients and $53 \%$ of the SPT $(\mathrm{P}>0.5)$. Urinary incontinence was seen in $12 \%$ of patients for both groups $(\mathrm{P}>0.5)$. In conclusion, the authors advocated the use of SPT, as a majority of patients undergoing PR required urethroplasty after development of impassable strictures.

A 2017 retrospective study, conducted by Horiguchi $e t$ al. evaluated 63 patients already treated with either SPT $(\mathrm{n}=49)$ vs. PR $(\mathrm{n}=14)$ and developed recurrent strictures. PR was assigned to any individual that had a urethral catheter inserted blindly or under endoscopic guidance. SPT was assigned to patients that either failed passage of a catheter or were initially treated with SPT. Patients 
were referred to their institution for delayed urethroplasty between 2008-2015 (33). The authors analyzed patients by stricture recurrence, types of procedures, erectile dysfunction, incontinence, and perioperative outcomes. False passage and iatrogenic scarring were evaluated by retrograde and antegrade urethrogram or magnetic resonance imaging. The mean time to urethroplasty in the PR group was 3 times longer than in the SPT group (133.1 vs. 47.0 months, $\mathrm{P}=0.035)$. Fifty percent of the patients in the PR group had repeat transurethral procedures such as urethrotomy, dilation, or urethral stenting prior to referral for urethroplasty and this was statistically significant when compared to the SPT group [10/49 (20.4\%), $\mathrm{P}=0.027]$. False passage and iatrogenic scars were significantly higher $(\mathrm{P}=0.035)$ in the $\mathrm{PR}$ group $(8 / 49,16.3 \%)$ vs. the SPT group $(6 / 14,42.9 \%)$. In regards to urethroplasty outcomes, the success rate was slightly higher in the SPT group (45/49, $91.8 \%)$ compared to the PR group (12/14, 85.7\%) but this was not statistically significant. In considering only the 46 patients who had no history of prior transurethral treatment, their results were statistically significant in favor of SPT having a higher success rate compared to the PR group $(37 / 39,94.9 \%$ vs. $5 / 7,71.4 \%$; $\mathrm{P}=0.042)$. Six months post-operatively, 38 patients were available for evaluation of incontinence and erectile dysfunction. Incontinence was assessed based on the need for any protective pads. Erectile dysfunction was defined as a score of less than 21 on the International Index of Erectile Function. There was no statistical difference in the incidence of incontinence (20\% SPT vs. $25 \% \mathrm{PR} ; \mathrm{P}=0.757$ ) or erectile dysfunction (76.7\% SPT vs. $62.5 \%$ PR; $\mathrm{P}=0.418$ ) between management strategies. Therefore, the authors concluded that patients who undergo PR are at an increased risk of complicated stricture and longer disease duration as a result of increased need for repeated transurethral procedures.

\section{Level 4 studies}

Historically, the evaluation of PFUI management options has focused solely on the urological outcomes; with little attention paid to the consequences these management options might have on concomitant orthopedic injuries. A case-series consisting of eight patients conducted at University of Tennessee, evaluated how the urological management of posterior urethral injuries conflicted with coexisting pelvic fracture management (34). Although not statistically significant, it was also noted that patients who were treated with SPT had a longer length of immobilization compared to those who underwent realignment (mean 39 vs. 7 days; $\mathrm{P}=0.17$ ) and average length of hospital stay ( 31 vs. 18 days; $\mathrm{P}=0.37$ ). Of the 4 patients who were managed with SPT and therefore received no pelvic surgery, 3 ( $75 \%$ ) remained disabled. None of those who underwent PER remained disabled because of their pelvic fracture.

\section{Summary on suprapubic cystostomy and delayed repair}

Until the advent of PER, suprapubic cystostomy with delayed repair was perhaps considered the most dependable option for managing a patient with a PFUI with minimal morbidity. However, there is a paucity of data demonstrating superiority of SPT alone for patients with urethral disruption. Based on the current data available $83-100 \%$ of develop strictures, $0-25 \%$ incontinence, and $16.2-90.1 \%$ erectile dysfunction. See Table 1 for a summary. Limitations of the current studies include: all were retrospective in nature, in some studies, SPT placement was performed at referring hospitals and thus the true number of patients undergoing SPT alone cannot be determined, lack of validated questionnaires for erectile dysfunction and incontinence, and several studies treated patients with SPT only after failed PER indicating a possibly more severe urethral injury. Nevertheless, based on the current literature available, it appears that SPT might be the best option available for patients who are clinically unstable or for a urologist who is unfamiliar with PER. Urologists, who decide to manage their patients with SPT, can then allow the patient to recover from their injuries, and refer them later on to a specialist, or if qualified, perform delayed urethroplasty.

\section{Conclusions}

The management of acute PFUI remains a highly debated topic as currently there is no level I evidence. Over the past 3 decades, retrospective studies have illustrated the possible benefits of PER over urinary diversion with SPT, mostly noting the reduced need for complex urethroplasty. However, aside from a level 2 study done by Hadjizacharia et al., no prospective study has evaluated the potential of PER to be a superior management to SPT in preventing urethral stricture, erectile dysfunction, urinary incontinence, and need for urethroplasty. In effort to answer this question, the American Association for the Surgery of Trauma, is enrolling patients in a prospective multi-institutional cohort study to 
evaluate the incidence of urethral obstruction in patients randomized with either SPT vs. PER. This study will be the largest level 2 study to date and will elucidate superiority of either management strategy for patients with PFUI.

\section{Acknowledgements}

None.

\section{Footnote}

Conflicts of Interest: The authors have no conflicts of interest to declare.

\section{References}

1. Brandes S. Initial management of anterior and posterior urethral injuries. Urol Clin North Am 2006;33:87-95, vii.

2. Pokorny M, Pontes JE, Pierce JM Jr. Urological injuries associated with pelvic trauma. J Urol 1979;121:455-7.

3. Guillé F, Cippola B, el Khader K, et al. Early endoscopic realignment for complete traumatic rupture of the posterior urethra--21 patients. Acta Urol Belg 1998;66:55-8.

4. Basta AM, Blackmore CC, Wessells H. Predicting urethral injury from pelvic fracture patterns in male patients with blunt trauma. J Urol 2007;177:571-5.

5. Figler BD, Hoffler CE, Reisman W, et al. Multidisciplinary update on pelvic fracture associated bladder and urethral injuries. Injury 2012;43:1242-9.

6. Flaherty JJ, Kelley R, Burnett B, et al. Relationship of pelvic bone fracture patterns to injuries of urethra and bladder. J Urol 1968;99:297-300.

7. Lynch TH, Martínez-Piñeiro L, Plas E, et al. EAU guidelines on urological trauma. Eur Urol 2005;47:1-15.

8. Morey AF, Brandes S, Dugi DD 3rd, et al. Urotrauma: AUA guideline. J Urol 2014;192:327-35.

9. Chung KC, Swanson JA, Schmitz D, et al. Introducing evidence-based medicine to plastic and reconstructive surgery. Plast Reconstr Surg 2009;123:1385-9.

10. Ormond JK, Cothran RM. A Simple method of treating complete severance of the urethra complicating fracture of the pelvis. JAMA 1934;102:2180-1.

11. Wilkinson FO. Rupture of the posterior urethra with a review of twelve cases. Lancet 1961;1:1125-9.

12. Webster GD, Mathes GL, Selli C. Prostatomembranous urethral injuries: a review of the literature and a rational approach to their management. J Urol 1983;130:898-902.

13. Elliott DS, Barrett DM. Long-term followup and evaluation of primary realignment of posterior urethral disruptions. J Urol 1997;157:814-6.

14. DeWeerd JH. Immediate realignment of posterior urethral injury. Urol Clin North Am 1977;4:75-80.

15. Follis HW, Koch MO, McDougal WS. Immediate management of prostatomembranous urethral disruptions. J Urol 1992;147:1259-62.

16. Hadjizacharia P, Inaba K, Teixeira PG, et al. Evaluation of immediate endoscopic realignment as a treatment modality for traumatic urethral injuries. J Trauma 2008;64:1443-9; discussion 1449-50.

17. Tausch TJ, Morey AF. The case against primary endoscopic realignment of pelvic fracture urethral injuries. Arab J Urol 2015;13:13-6.

18. Zou Q, Zhou S, Zhang K, et al. The Immediate Management of Pelvic Fracture Urethral InjuryEndoscopic Realignment or Cystostomy? J Urol 2017;198:869-74.

19. Johnsen NV, Penson DF, Reynolds WS, et al. Costeffective management of pelvic fracture urethral injuries. World J Urol 2017;35:1617-23.

20. Chang PC, Hsu YC, Shee JJ, et al. Early endoscopic primary realignment decreases stricture formation and reduces medical costs in traumatic complete posterior urethral disruption in a 2-year follow-up. Chang Gung Med J 2011;34:179-85.

21. Koraitim MM. Pelvic fracture urethral injuries: evaluation of various methods of management. J Urol 1996;156:1288-91.

22. Asci R, Sarikaya S, Büyükalpelli R, et al. Voiding and sexual dysfunctions after pelvic fracture urethral injuries treated with either initial cystostomy and delayed urethroplasty or immediate primary urethral realignment. Scand J Urol Nephrol 1999;33:228-33.

23. Mouraviev VB, Coburn M, Santucci RA. The treatment of posterior urethral disruption associated with pelvic fractures: comparative experience of early realignment versus delayed urethroplasty. J Urol 2005;173:873-6.

24. Johnsen NV, Dmochowski RR, Mock S, et al. Primary Endoscopic Realignment of Urethral Disruption Injuries-A Double-Edged Sword? J Urol 2015;194:1022-6.

25. Barrett K, Braga LH, Farrokhyar F, et al. Primary realignment vs suprapubic cystostomy for the management of pelvic fracture-associated urethral injuries: a systematic review and meta-analysis. Urology 2014;83:924-9.

26. Koraitim MM. Effect of early realignment on length and delayed repair of postpelvic fracture urethral injury. Urology 2012;79:912-5.

27. Leddy LS, Vanni AJ, Wessells H, et al. Outcomes of 
endoscopic realignment of pelvic fracture associated urethral injuries at a level 1 trauma center. J Urol 2012;188:174-8.

28. Moudouni SM, Patard JJ, Manunta A, et al. Early endoscopic realignment of post-traumatic posterior urethral disruption. Urology 2001;57:628-32.

29. Johanson B. The reconstruction in stenosis of the male urethra. Z Urol 1953;46:361-75.

30. Morehouse DD. Management of posterior urethral rupture: a personal view. Br J Urol 1988;61:375-81.

31. Tausch TJ, Morey AF, Scott JF, et al. Unintended negative consequences of primary endoscopic realignment for men with pelvic fracture urethral injuries. J Urol

Cite this article as: Dixon AN, Webb JC, Wenzel JL, Wolf JS Jr, Osterberg EC. Current management of pelvic fracture urethral injuries: to realign or not? Transl Androl Urol 2018;7(4):593-602. doi: 10.21037/tau.2018.01.14
2014;192:1720-4.

32. Husmann DA, Wilson WT, Boone TB, et al. Prostatomembranous urethral disruptions: management by suprapubic cystostomy and delayed urethroplasty. J Urol 1990;144:76-8.

33. Horiguchi A, Shinchi M, Masunaga A, et al. Primary Realignment for Pelvic Fracture Urethral Injury Is Associated With Prolonged Time to Urethroplasty and Increased Stenosis Complexity. Urology 2017;108:184-9.

34. Mayher BE, Guyton JL, Gingrich JR. Impact of urethral injury management on the treatment and outcome of concurrent pelvic fractures. Urology 2001;57:439-42. 